PROCEEDINGS OF THE

AMERICAN MATHEMATICAL SOCIETY

Volume 125, Number 6, June 1997, Pages 1727-1735

S 0002-9939(97)03762-3

\title{
WEIGHTED HARDY-LITTLEWOOD INEQUALITY FOR $A$-HARMONIC TENSORS
}

\author{
SHUSEN DING
}

(Communicated by Theodore W. Gamelin)

\begin{abstract}
In this paper we prove a local weighted integral inequality for conjugate $A$-harmonic tensors similar to the Hardy and Littlewood integral inequality for conjugate harmonic functions. Then by using the local weighted integral inequality, we prove a global weighted integral inequality for conjugate $A$-harmonic tensors in John domains.
\end{abstract}

\section{INTRODUCTION AND NOTATION}

Conjugate harmonic functions have wide applications in many fields, such as potential theory, harmonic analysis and the theory of $H^{p}$-spaces. Conjugate $A$ harmonic tensors are interesting and important generalizations of conjugate harmonic functions and $p$-harmonic functions, $p>1$. Many interesting results of conjugate $A$-harmonic tensors and their applications in fields such as quasiregular mappings and the theory of elasticity have been found recently, see [N3], [I], [IM], $[\mathrm{S}],[\mathrm{B}]$ and $[\mathrm{BM}]$. In this paper, we prove local weighted inequalities and global weighted integral inequalities for conjugate $A$-harmonic tensors in John domains.

Let $e_{1}, e_{2}, \cdots, e_{n}$ denote the standard unit basis of $\mathbf{R}^{n}$. For $l=0,1, \cdots, n$, the linear space of $l$-vectors, spanned by the exterior products $e_{I}=e_{i_{1}} \wedge e_{i_{2}} \wedge \cdots e_{i_{l}}$, corresponding to all ordered $l$-tuples $I=\left(i_{1}, i_{2}, \cdots, i_{l}\right), 1 \leq i_{1}<i_{2}<\cdots<i_{l} \leq n$, is denoted by $\wedge^{l}=\wedge^{l}\left(\mathbf{R}^{n}\right)$. The Grassmann algebra $\wedge=\oplus \wedge^{l}$ is a graded algebra with respect to the exterior products. For $\alpha=\sum \alpha^{I} e_{I} \in \wedge$ and $\beta=\sum \beta^{I} e_{I} \in \wedge$, the inner product in $\wedge$ is given by

$$
\langle\alpha, \beta\rangle=\sum \alpha^{I} \beta^{I}
$$

with summation over all $l$-tuples $I=\left(i_{1}, i_{2}, \cdots, i_{l}\right)$ and all integers $l=0,1, \cdots, n$. We define the Hodge star operator $\star: \wedge \rightarrow \wedge$ by the rule

$$
\star 1=e_{1} \wedge e_{2} \wedge \cdots \wedge e_{n} \quad \text { and } \quad \alpha \wedge \star \beta=\beta \wedge \star \alpha=\langle\alpha, \beta\rangle(\star 1)
$$

for all $\alpha, \beta \in \wedge$.

Hence the norm of $\alpha \in \wedge$ is given by the formula $|\alpha|^{2}=\langle\alpha, \alpha\rangle=\star(\alpha \wedge \star \alpha) \in$ $\wedge^{0}=\mathbf{R}$. The Hodge star is an isometric isomorphism on $\wedge$ with $\star: \wedge^{l} \rightarrow \wedge^{n-l}$ and $\star \star(-1)^{l(n-l)}: \wedge^{l} \rightarrow \wedge^{l}$.

Received by the editors May 15, 1995 and, in revised form, December 8, 1995.

1991 Mathematics Subject Classification. Primary 30C65; Secondary 31B05, 58A10.

Key words and phrases. Conjugate harmonic tensors, differential forms and the $A$-harmonic equation. 
Throughout this paper, we always assume $\Omega$ is a connected open subset of $\mathbf{R}^{n}$. We write $\mathbf{R}=\mathbf{R}^{1}$. Cubes or balls are denoted by $Q$ and $\sigma Q$ is the cube or ball with the same center as $Q$ and with $\operatorname{diam}(\sigma Q)=\sigma \operatorname{diam}(Q)$. The $n$-dimensional Lebesgue measure of a set $E \subseteq \mathbf{R}^{n}$ is denoted by $|E|$. Suppose that $w \in L_{l o c}^{1}\left(\mathbf{R}^{n}\right)$, $w>0$ a.e., and $0<p<\infty$. We denote the weighted $L^{p}$-norm of a measurable function $f$ over $E$ by

$$
\|f\|_{p, E, w}=\left(\int_{E}|f(x)|^{p} w(x) d x\right)^{1 / p} .
$$

A differential $l$-form $\omega$ on $\Omega$ is a Schwartz distribution on $\Omega$ with values in $\wedge^{l}\left(\mathbf{R}^{n}\right)$. We denote the space of differential $l$-forms by $D^{\prime}\left(\Omega, \wedge^{l}\right)$. We write $L^{p}\left(\Omega, \wedge^{l}\right)$ for the $l$-forms $\omega(x)=\sum_{I} \omega_{I}(x) d x_{I}=\sum \omega_{i_{1} i_{2} \cdots i_{l}}(x) d x_{i_{1}} \wedge d x_{i_{2}} \wedge \cdots \wedge d x_{i_{l}}$ with $\omega_{I} \in$ $L^{p}(\Omega, \mathbf{R})$ for all ordered $l$-tuples $I$. Thus $L^{p}\left(\Omega, \wedge^{l}\right)$ is a Banach space with norm

$$
\|\omega\|_{p, \Omega}=\left(\int_{\Omega}|\omega(x)|^{p} d x\right)^{1 / p}=\left(\int_{\Omega}\left(\sum_{I}\left|\omega_{I}(x)\right|^{2}\right)^{p / 2} d x\right)^{1 / p} .
$$

Similarly, $W_{p}^{1}\left(\Omega, \wedge^{l}\right)$ are those differential $l$-forms on $\Omega$ whose coefficients are in $W_{p}^{1}(\Omega, \mathbf{R})$. The notations $W_{p, l o c}^{1}(\Omega, \mathbf{R})$ and $W_{p, l o c}^{1}\left(\Omega, \wedge^{l}\right)$ are self-explanatory. We denote the exterior derivative by $d: D^{\prime}\left(\Omega, \wedge^{l}\right) \rightarrow D^{\prime}\left(\Omega, \wedge^{l+1}\right)$ for $l=0,1, \cdots, n$. Its formal adjoint operator $d^{\star}: D^{\prime}\left(\Omega, \wedge^{l+1}\right) \rightarrow D^{\prime}\left(\Omega, \wedge^{l}\right)$ is given by $d^{\star}=$ $(-1)^{n l+1} \star d \star \quad$ on $D^{\prime}\left(\Omega, \wedge^{l+1}\right), l=0,1, \cdots, n$.

Definition 1.1. We call $u$ a $p$-harmonic function if $u$ satisfies the $p$-harmonic equation

$$
\operatorname{div}\left(\nabla u|\nabla u|^{p-2}\right)=0
$$

with $p>1$. Its conjugate in the plane is a $q$-harmonic function $v, p^{-1}+q^{-1}=1$, which satisfies

$$
\nabla u|\nabla u|^{p-2}=\left(\frac{\partial v}{\partial y},-\frac{\partial v}{\partial x}\right)
$$

Note that if $p=q=2$, we get the usual conjugate harmonic funtions. If $\omega: \Omega \rightarrow \wedge^{l}$, then the value of $\omega(x)$ at the vectors $\xi_{1}, \cdots, \xi_{l} \in \mathbf{R}^{n}$ will be denoted by $\omega\left(x ; \xi_{1}, \cdots, \xi_{l}\right)$. The following lemma appears in [IL].

Lemma 1.3. Let $Q \subset \mathbf{R}^{n}$ be a cube. To each $y \in Q$ there corresponds a linear operator $K_{y}: C^{\infty}\left(Q, \wedge^{l}\right) \rightarrow C^{\infty}\left(Q, \wedge^{l-1}\right)$ defined by

$$
\left(K_{y} \omega\right)\left(x ; \xi_{1}, \cdots, \xi_{l}\right)=\int_{0}^{1} t^{l-1} \omega\left(t x+y-t y ; x-y, \xi_{1}, \cdots, \xi_{l-1}\right) d t
$$

and the decomposition

$$
\omega=d\left(K_{y} \omega\right)+K_{y}(d \omega)
$$

We define another linear operator $T_{Q}: C^{\infty}\left(Q, \wedge^{l}\right) \rightarrow C^{\infty}\left(Q, \wedge^{l-1}\right)$ by averaging $K_{y}$ over points $y$ in $Q$

$$
T_{Q} \omega=\int_{Q} \varphi(y) K_{y} \omega d y
$$


where $\varphi \in C_{0}^{\infty}(Q)$ is normalized by $\int_{Q} \varphi(y) d y=1$. We define the $l$-form $\omega_{Q} \in$ $D^{\prime}\left(Q, \wedge^{l}\right)$ by

$$
\omega_{Q}=|Q|^{-1} \int_{Q} \omega(y) d y, \quad l=0, \text { and } \omega_{Q}=d\left(T_{Q} \omega\right), \quad l=1,2, \cdots, n,
$$

for all $\omega \in L^{p}\left(Q, \wedge^{l}\right), 1 \leq p<\infty$.

In recent years there has been new interest developed in the study of the $A$ harmonic equation for differential forms, largely pertaining to applications in quasiconformal analysis and nonlinear elasticity, that is:

$$
d^{\star} A(x, d \omega)=0,
$$

where $A: \Omega \times \wedge^{l}\left(\mathbf{R}^{n}\right) \rightarrow \wedge^{l}\left(\mathbf{R}^{n}\right)$ satisfies the following conditions:

$$
|A(x, \xi)| \leq a|\xi|^{p-1} \text { and }\langle A(x, \xi), \xi\rangle \geq|\xi|^{p}
$$

for almost every $x \in \Omega$ and all $\xi \in \wedge^{l}\left(\mathbf{R}^{n}\right)$. Here $a>0$ is a constant and $1<p<\infty$ is a fixed exponent associated with (1.6). A solution to (1.6) is an element of the Sobolev space $W_{p, l o c}^{1}\left(\Omega, \wedge^{l-1}\right)$ such that

$$
\int_{\Omega}\langle A(x, d \omega), d \varphi\rangle=0
$$

for all $\varphi \in W_{p}^{1}\left(\Omega, \wedge^{l-1}\right)$ with compact support.

In order to formulate the Hardy-Littlewood type estimate it is required first of all that the equation is written in the form of a first order differential system:

$$
A(x, d u)=d^{\star} v .
$$

In this way we obtain a pair $(u, v)$ of $(l-1)$-form $u$ and $(l+1)$-form $v$, called the conjugate $A$-harmonic fields. Example: $d u=d^{*} v$ is an analogue of a CauchyRiemann system in $\mathbf{R}^{n}$. Clearly, the $A$-harmonic equation is not affected by adding a closed form to $u$ and coclosed form to $v$. Therefore, any type of estimates between $u$ and $v$ must be modulo such forms. Suppose that $u$ is a solution to (1.6) in $\Omega$. Then by Poincaré's lemma, at least locally in a ball $B$, there exists a form $v \in W_{q}^{1}\left(B, \wedge^{l+1}\right), \frac{1}{p}+\frac{1}{q}=1$, such that (1.8) holds.

Definition 1.9. When $u$ and $v$ satisfy (1.8) in $\Omega$, and $A^{-1}$ exists in $\Omega$, we call $u$ and $v$ conjugate $A$-harmonic tensors in $\Omega$.

Hardy and Littlewood in [HL] proved the following result.

Theorem A. For each $p>0$, there is a constant $C$ such that

$$
\int_{D}|u-u(0)|^{p} d x d y \leq C \int_{D}|v-v(0)|^{p} d x d y
$$

for all analytic functions $f=u+i v$ in the unit disk $D$.

Craig A. Nolder proves the similar results about $K$-quasiregular mappings in [N1] and [N2]. Recently, Craig A. Nolder generalized the above result and proved the following important Theorem B and Theorem $\mathrm{C}$ about conjugate $A$-harmonic tensors [N3]. 
Theorem B. Let $u$ and $v$ be conjugate A-harmonic tensors in $\Omega \subset \mathbf{R}^{n}, \sigma>1$, and $0<s, t<\infty$. Then there exists a constant $C$, independent of $u$ and $v$, such that

$$
\left\|u-u_{Q}\right\|_{s, Q} \leq C|Q|^{\beta}\left\|v-c_{1}\right\|_{t, \sigma Q}^{q / p}
$$

and

$$
\left\|v-v_{Q}\right\|_{t, Q} \leq C|Q|^{-\beta p / q}\left\|u-c_{2}\right\|_{s, \sigma Q}^{p / q}
$$

for all cubes $Q$ with $\sigma Q \subset \Omega$. Here $c_{1}$ is any form in $W_{p, l o c}^{1}(\Omega, \Lambda)$ with $d^{\star} c_{1}=0$, $c_{2}$ is any form in $W_{q, l o c}^{1}(\Omega, \Lambda)$ with $d c_{2}=0$ and $\beta=1 / s+1 / n-(1 / t+1 / n) q / p$.

Theorem C. Let $u \in D^{\prime}\left(\Omega, \Lambda^{0}\right)$ and $v \in D^{\prime}\left(\Omega, \Lambda^{2}\right)$ be conjugate A-harmonic tensors and $0<s, t<\infty$. If $\Omega$ is a $\delta$-John domain, $q \leq p, v-c \in L^{t}\left(\Omega, \Lambda^{2}\right)$ and

$$
s=\Phi(t)=\frac{n p t}{n q+t(q-p)}, \quad 0<t<\infty,
$$

then $u \in L^{s}\left(\Omega, \Lambda^{0}\right)$ and moreover, there exists a constant $C$, independent of $u$ and $v$, such that

$$
\left\|u-u_{Q_{0}}\right\|_{s, \Omega} \leq C\|v-c\|_{t, \Omega}^{q / p} .
$$

Here $c$ is any form in $W_{q, l o c}^{1}(\Omega, \Lambda)$ with $d^{*} c=0$.

Our main results Theorem 2.4 and Theorem 3.4 generalize (1.10) and (1.12), respectively.

\section{The LOCAL WEIGHTED INTEGRAL INEQUALITY}

We will use the following generalized Hölder's inequality.

Lemma 2.1. Let $0<\alpha<\infty, 0<\beta<\infty$ and $s^{-1}=\alpha^{-1}+\beta^{-1}$. If $f$ and $g$ are measurable functions on $\mathbf{R}^{n}$, then

$$
\|f g\|_{s, \Omega} \leq\|f\|_{\alpha, \Omega} \cdot\|g\|_{\beta, \Omega}
$$

for any $\Omega \subset \mathbf{R}^{n}$.

The following definition appears in $[\mathrm{G}]$.

Definition 2.2. We say the weight $w(x)>0$ satisfies the $A_{r}$ condition, $r>1$, write $w \in A_{r}$, if

$$
\sup _{Q}\left(\frac{1}{|Q|} \int_{Q} w d x\right)\left(\frac{1}{|Q|} \int_{Q}\left(\frac{1}{w}\right)^{1 /(r-1)} d x\right)^{r-1}<\infty
$$

for any cube $Q \subset \mathbf{R}^{n}$.

We also need the following lemma $[\mathrm{G}]$.

Lemma 2.3. If $w \in A_{r}, r>1$, then there exist constants $\beta>1$ and $C$, independent of $w$, such that

$$
\|w\|_{\beta, Q} \leq C|q|^{(1-\beta) / \beta}\|w\|_{1, Q}
$$

for all cubes $Q \subset \mathbf{R}^{n}$

Now, we can prove the following local weighted result. 
Theorem 2.4. Let $u$ and $v$ be conjugate A-harmonic tensors in a domain $\Omega \subset \mathbf{R}^{n}$ and $w \in A_{r}$. Let $s=\Phi(t)$ be defined by (1.11). Then there exists a constant $C$, independent of $u$ and $v$, such that

$$
\left(\int_{Q}\left|u-u_{Q}\right|^{s} w d x\right)^{1 / s} \leq C\left(\int_{\sigma Q}|v-c|^{t} w^{p t / q s} d x\right)^{q / p t}
$$

for all cubes $Q$ with $\sigma Q \subset \Omega \subset \mathbf{R}^{n}$ and $\sigma>1$. Here $c$ is any form in $W_{q, l o c}^{1}(\Omega, \Lambda)$ with $d^{*} c=0$.

Proof. By Lemma 2.3, there exist constants $\alpha>1$ and $C_{1}$, independent of $w$, such that

$$
\|w\|_{\alpha, \sigma Q} \leq C_{1}|Q|^{(1-\alpha) / \alpha}\|w\|_{1, \sigma Q} .
$$

Since $1 / \alpha s+(\alpha-1) / \alpha s=1 / s$, then by Lemma 2.1 , we have

$$
\left\|u-u_{Q}\right\|_{s, Q, w} \leq\|w\|_{\alpha, Q}^{1 / s} \cdot\left\|u-u_{Q}\right\|_{\alpha s /(\alpha-1), Q} .
$$

By Theorem $\mathrm{B}$, there is a constant $C_{2}$, independent of $u$ and $v$, such that for any $t^{\prime}>0$, we have

$$
\left\|u-u_{Q}\right\|_{\alpha s /(\alpha-1), Q} \leq C_{2}|Q|^{\beta^{\prime}} \cdot\|v-c\|_{t^{\prime}, \sigma Q}^{q / p},
$$

where $\beta^{\prime}=(\alpha-1) / \alpha s+1 / n-\left(1 / t^{\prime}+1 / n\right) q / p$. Combining (2.7) and (2.8), we obtain

$$
\left\|u-u_{Q}\right\|_{s, Q, w} \leq C_{2}|Q|^{\beta^{\prime}} \cdot\|w\|_{\alpha, Q}^{1 / s} \cdot\|v-c\|_{t^{\prime}, \sigma Q}^{q / p} .
$$

Now, choose $t^{\prime}=t / k$, where $k$ is to be determined later, and note $|v-c|=$ $w^{-p / q s}|v-c| w^{p / q s}$; by Lemma 2.1 , we get

$$
\|v-c\|_{t^{\prime}, \sigma Q} \leq\left\|(1 / w)^{p t / q s}\right\|_{1 /(k-1), \sigma Q}^{1 / t} \cdot\left(\int_{\sigma Q}|v-c|^{t} w^{p t / q s} d x\right)^{1 / t} .
$$

From (2.6), (2.9) and (2.10) we have

$$
\begin{aligned}
\left\|u-u_{Q}\right\|_{s, Q, w} \leq & C_{3}|Q|^{\beta^{\prime}+(1-\alpha) / \alpha s}\|w\|_{1, \sigma Q}^{1 / s} \\
& \cdot\left\|\left(\frac{1}{w}\right)^{p t / q s}\right\|_{1 /(k-1), \sigma Q}^{q / p t}\left(\int_{\sigma Q}|v-c|^{t} w^{p t / q s} d x\right)^{q / p t} .
\end{aligned}
$$

We choose $k=1+p t(r-1) / q s$. Then $(k-1) q s / p t=r-1$, and by $w \in A_{r}$, we know

$$
\begin{aligned}
\| & w\left\|_{1, \sigma Q}^{1 / s}\right\|\left(\frac{1}{w}\right)^{p t / q s} \|_{1 /(k-1), \sigma Q}^{q / p t} \\
& =|\sigma Q|^{1 / s+(k-1) q / p t}\left(\frac{1}{|\sigma Q|} \int_{\sigma Q} w d x\left(\frac{1}{|\sigma Q|} \int_{\sigma Q}\left(\frac{1}{w}\right)^{1 /(r-1)} d x\right)^{r-1}\right)^{1 / s} \\
& \leq C_{4}|Q|^{1 / s+(k-1) q / p t} .
\end{aligned}
$$

By (2.11) and (2.12), we have

$$
\left\|u-u_{Q}\right\|_{s, Q, w} \leq C_{5}|Q|^{\gamma}\left(\int_{\sigma Q}|v-c|^{t} w^{p t / q s} d x\right)^{q / p t}
$$


where $\gamma=\beta^{\prime}+(1-\alpha) / \alpha s+1 / s+q(k-1) / p t=-(n q+t(q-p)) / n p t+1 / s=0$ by (1.11). So (2.13) becomes

$$
\left\|u-u_{Q}\right\|_{s, Q, w} \leq C\left(\int_{\sigma Q}|v-c|^{t} w^{p t / q s} d x\right)^{q / p t},
$$

that is,

$$
\left(\int_{Q}\left|u-u_{Q}\right|^{s} w d x\right)^{1 / s} \leq C\left(\int_{\sigma Q}|v-c|^{t} w^{p t / q s} d x\right)^{q / p t} .
$$

We have completed the proof of Theorem 2.4.

\section{The GLOBAL WEIGHTED INTEGRAL INEQUALITY}

Definition 3.1. We call $\Omega$, a proper subdomain of $\mathbf{R}^{n}$, a $\delta$-John domain, $\delta>0$, if there exists a point $x_{0} \in \Omega$ which can be joined with any other point $x \in \Omega$ by a continuous curve $\gamma \subset \Omega$ so that

$$
d(\xi, \partial \Omega) \geq \delta|x-\xi|
$$

for each $\xi \in \gamma$. Here $d(\xi, \partial \Omega)$ is the Euclidean distance between $\xi$ and $\partial \Omega$.

We know that a $\delta$-John domain has the following properties [N3].

Lemma 3.2. Let $\Omega \subset \mathbf{R}^{n}$ be a $\delta$-John domain. Then there exists a covering $\mathcal{V}$ of $\Omega$ consisting of open cubes such that:

i) $\sum_{Q \in \mathcal{V}} \chi_{\sigma Q}(x) \leq N \chi_{\Omega}(x), \quad x \in \mathbf{R}^{n}$.

ii) There is a distinguished cube $Q_{0} \in \mathcal{V}$ (called the central cube) which can be connected with every cube $Q \in \mathcal{V}$ by a chain of cubes $Q_{0}, Q_{1}, \cdots, Q_{k}=Q$ from $\mathcal{V}$ such that for each $i=0,1, \cdots, k-1$,

$$
Q \subset N Q_{i}
$$

There is a cube $R_{i} \subset \mathbf{R}^{n}$ (this cube does not need be a member of $\mathcal{V}$ ) such that

$$
R_{i} \subset Q_{i} \cap Q_{i+1} \text {, and } Q_{i} \cup Q_{i+1} \subset N R_{i} \text {. }
$$

The following lemma appears in [IN].

Lemma 3.3. If $\mathcal{V}$ is a collection of cubes in $\mathbf{R}^{n}$ and $C_{Q}$ are non-negative numbers associated with the cubes $Q \in \mathcal{V}$ and $w \in A_{r}, d \mu(x)=w(x) d x$, then for $1 \leq p<\infty$ and $N \geq 1$ we have

$$
\left(\int_{\mathbf{R}^{n}}\left(\sum_{Q \in \mathcal{V}} C_{Q} \cdot \chi_{N Q}\right)^{p} d \mu(x)\right)^{1 / p} \leq B_{p}\left(\int_{\mathbf{R}^{n}}\left(\sum_{Q \in \mathcal{V}} C_{Q} \cdot \chi_{Q}\right)^{p} d \mu(x)\right)^{1 / p},
$$

where $B_{p}$ is independent of the collection $\mathcal{V}$ and the numbers $C_{Q}$.

Theorem 3.4. Let $u \in D^{\prime}\left(\Omega, \Lambda^{0}\right)$ and $v \in D^{\prime}\left(\Omega, \Lambda^{2}\right)$ be conjugate A-harmonic tensors. Let $q \leq p, v-c \in L^{t}\left(\Omega, \Lambda^{2}\right), w \in A_{r}$ and $s=\Phi(t)$ is defined in (1.11). Then there exists a constant $C$, independent of $u$ and $v$, such that

$$
\left(\int_{\Omega}\left|u-u_{Q_{0}}\right|^{s} w d x\right)^{1 / s} \leq C\left(\int_{\Omega}|v-c|^{t} w^{p t / q s} d x\right)^{q / p t}
$$

for any $\delta$-John domain $\Omega \subset \mathbf{R}^{n}$. Here $c$ is any form in $W_{q, l o c}^{1}(\Omega, \Lambda)$ with $d^{*} c=0$ and $Q_{0} \subset \Omega$ is the cube appearing in Lemma 3.2 . 
Proof. Since $w \in A_{r}$, we can write $d \mu(x)=w(x) d x$; then (2.5) can be written as

$$
\int_{Q}\left|u-u_{Q}\right|^{s} d \mu(x) \leq C\left(\int_{\sigma Q}|v-c|^{t} w^{p t / q s} d x\right)^{q s / p t} .
$$

We use the notations and the covering $\mathcal{V}$ described in the above Lemma 3.2 and the properties of the measure $d \mu(x)=w(x) d x$ : if $w \in A_{r}$, then

$$
\mu(N Q) \leq M N^{n r} \mu(Q)
$$

for each cube $Q$ with $N Q \subset \mathbf{R}^{n}$ (see [G]) and

$$
\max \left(\mu\left(Q_{i}\right), \mu\left(Q_{i+1}\right)\right) \leq M N^{n r} \mu\left(Q_{i} \cap Q_{i+1}\right)
$$

for the sequence of cubes $Q_{i}, Q_{i+1}, i=0,1, \cdots, k-1$ described in ii). We will use the elementary inequality $|a+b|^{s} \leq 2^{s}\left(|a|^{s}+|b|^{s}\right)$ for all $s>0$. In particular we have

$$
\begin{aligned}
\int_{\Omega}\left|u-u_{Q_{0}}\right|^{s} w d x & =\int_{\Omega}\left|u-u_{Q_{0}}\right|^{s} d \mu(x) \\
& \leq 2^{s} \sum_{Q \in \mathcal{V}} \int_{Q}\left|u-u_{Q}\right|^{s} d \mu(x)+2^{s} \sum_{Q \in \mathcal{V}} \int_{Q}\left|u_{Q_{0}}-u_{Q}\right|^{s} d \mu(x) .
\end{aligned}
$$

The first sum can be estimated by (3.5) and the condition i):

$$
\begin{aligned}
\sum_{Q \in \mathcal{V}} \int_{Q}\left|u-u_{Q}\right|^{s} d \mu(x) & \leq C_{1} \sum_{Q \in \mathcal{V}}\left(\int_{\sigma Q}|v-c|^{t} w^{p t / q s} d x\right)^{q s / p t} \\
& \leq C_{1} N\left(\int_{\Omega}|v-c|^{t} w^{p t / q s} d x\right)^{q s / p t} .
\end{aligned}
$$

Now we estimate the second sum in (3.8). Fix a cube $Q \in \mathcal{V}$ and let $Q_{0}, Q_{1}, \cdots$, $Q_{k}=Q$ be the chain from ii). We have

$$
\left|u_{Q_{0}}-u_{Q}\right| \leq \sum_{i=0}^{k-1}\left|u_{Q_{i}}-u_{Q_{i+1}}\right| .
$$

From (3.5) and (3.7) we have

$$
\begin{aligned}
\left|u_{Q_{i}}-u_{Q_{i+1}}\right|^{s} & =\frac{1}{\mu\left(Q_{i} \cap Q_{i+1}\right)} \int_{Q_{i} \cap Q_{i+1}}\left|u_{Q_{i}}-u_{Q_{i+1}}\right|^{s} d \mu(x) \\
& \leq \frac{M N^{n r}}{\max \left(\mu\left(Q_{i}\right), \mu\left(Q_{i+1}\right)\right)} \int_{Q_{i} \cap Q_{i+1}}\left|u_{Q_{i}}-u_{Q_{i+1}}\right|^{s} d \mu(x) \\
& \leq C_{2} \sum_{j=i}^{i+1} \frac{1}{\mu\left(Q_{j}\right)} \int_{Q_{j}}\left|u-u_{Q_{j}}\right|^{s} d \mu(x) \\
& \leq C_{3} \sum_{j=i}^{i+1} \frac{1}{\mu\left(Q_{j}\right)}\left(\int_{\sigma Q_{j}}|v-c|^{t} w^{p t / q s} d x\right)^{q s / p t}
\end{aligned}
$$

Since $Q \subset N Q_{j}$ for $j=i, i+1,0 \leq i \leq k-1$ (see ii)), we have

$$
\left|u_{Q_{i}}-u_{Q_{i+1}}\right|^{s} \chi_{Q}(x) \leq C_{3} \sum_{j=i}^{i+1} \frac{\chi_{N Q_{j}}(x)}{\mu\left(Q_{j}\right)}\left(\int_{\sigma Q_{j}}|v-c|^{t} w^{p t / q s} d x\right)^{q s / p t} .
$$


By (3.10) we have (note $|a+b|^{1 / s} \leq 2^{1 / s}\left(|a|^{1 / s}+|b|^{1 / s}\right)$ )

$$
\left|u_{Q_{0}}-u_{Q}\right| \chi_{Q}(x) \leq C_{4} \sum_{R \in \mathcal{V}}\left(\frac{1}{\mu(R)}\left(\int_{\sigma R}|v-c|^{t} w^{p t / q s} d x\right)^{q s / p t}\right)^{1 / s} \cdot \chi_{N R}(x)
$$

for every $x \in \mathbf{R}^{n}$. Hence

$$
\begin{aligned}
& \sum_{Q \in \mathcal{V}} \int_{Q}\left|u_{Q_{0}}-u_{Q}\right|^{s} d \mu(x) \\
& \quad \leq C_{5} \int_{R^{n}}\left|\sum_{R \in \mathcal{V}}\left(\frac{1}{\mu(R)}\left(\int_{\sigma R}|v-c|^{t} w^{p t / q s} d x\right)^{q s / p t}\right)^{1 / s} \chi_{N R}(x)\right|^{s} d \mu(x) .
\end{aligned}
$$

If $0 \leq s \leq 1$, we use the inequality $\left|\sum t_{\alpha}\right|^{s} \leq \sum\left|t_{\alpha}\right|^{s},(3.6)$ and the condition i) to get

$$
\begin{aligned}
\sum_{Q \in \mathcal{V}} \int_{Q}\left|u_{Q_{0}}-u_{Q}\right|^{s} d \mu(x) & \leq C_{6} \sum_{R \in \mathcal{V}} \frac{\mu(N R)}{\mu(R)}\left(\int_{\sigma R}|v-c|^{t} w^{p t / q s} d x\right)^{q s / p t} \\
& \leq C_{7} \sum_{R \in \mathcal{V}}\left(\int_{\sigma R}|v-c|^{t} w^{p t / q s} d x\right)^{q s / p t}
\end{aligned}
$$

Note $q s / p t \geq 1$ and $\sum t_{\alpha}^{p} \leq\left(\sum t_{\alpha}\right)^{p}$ for $p \geq 1$ and $t_{\alpha}>0$; then

$$
\begin{aligned}
\sum_{Q \in \mathcal{V}} \int_{Q}\left|u_{Q_{0}}-u_{Q}\right|^{s} d \mu(x) & \leq C_{7} \sum_{R \in \mathcal{V}}\left(\int_{\Omega}|v-c|^{t} w^{p t / q s} \chi_{\sigma R}(x) d x\right)^{q s / p t} \\
& \leq C_{7}\left(\int_{\Omega}|v-c|^{t} w^{p t / q s} \sum_{R \in \mathcal{V}} \chi_{\sigma R}(x) d x\right)^{q s / p t} \\
& \leq C_{7}\left(\int_{\Omega}|v-c|^{t} w^{p t / q s} \chi_{\Omega}(x) d x\right)^{q s / p t} \\
& \leq C_{8}\left(\int_{\Omega}|v-c|^{t} w^{p t / q s} d x\right)^{q s / p t} .
\end{aligned}
$$

Combining (3.8), (3.9) and (3.12), we have proved our theorem for the case $0<$ $s \leq 1$.

For the case $1 \leq s<\infty$, by (3.11) and Lemma 3.3, we have

$$
\begin{aligned}
& \sum_{Q \in \mathcal{V}} \int_{Q}\left|u_{Q_{0}}-u_{Q}\right|^{s} d \mu(x) \\
& \quad \leq C_{9} \int_{R^{n}}\left|\sum_{R \in \mathcal{V}}\left(\frac{1}{\mu(R)}\left(\int_{\sigma R}|v-c|^{t} w^{p t / q s} d x\right)^{q s / p t}\right)^{1 / s} \chi_{R}(x)\right|^{s} d \mu(x) .
\end{aligned}
$$

Note

$$
\sum_{R \in \mathcal{V}} \chi_{R}(x) \leq \sum_{R \in \mathcal{V}} \chi_{\sigma R}(x) \leq N \chi_{\Omega}(x)
$$


with the elementary inequality $\left|\sum_{i=1}^{N} t_{i}\right|^{s} \leq N^{s-1} \sum_{i=1}^{N}\left|t_{i}\right|^{s}$; we obtain

$$
\begin{aligned}
\sum_{Q \in \mathcal{V}} & \int_{Q}\left|u_{Q_{0}}-u_{Q}\right|^{s} d \mu(x) \\
& \leq C_{10} \int_{R^{n}}\left(\sum_{R \in \mathcal{V}} \frac{1}{\mu(R)}\left(\int_{\sigma R}|v-c|^{t} w^{p t / q s} d x\right)^{q s / p t} \chi_{R}(x)\right) d \mu(x) \\
& =C_{10} \sum_{R \in \mathcal{V}}\left(\int_{\sigma R}|v-c|^{t} w^{p t / q s} d x\right)^{q s / p t} \\
& \leq C_{11}\left(\int_{\Omega}|v-c|^{t} w^{p t / q s} d x\right)^{q s / p t}
\end{aligned}
$$

by the condition i). Combining (3.8), (3.9) and (3.13), we have proved the theorem for the case $1 \leq s<\infty$; thus, we have completed the proof of Theorem 3.4.

\section{ACKNOWLEDGMENTS}

I would like to thank Professor Craig A. Nolder for his advice and continuous encouragement in the preparation of this paper. The author also thanks the referee and the editor, Professor T.W. Gamelin, for their precious and thoughtful suggestions on this paper.

\section{REFERENCES}

[B] Ball, J. M., Convexity conditions and existence theorems in nonlinear elasticity, Arch. Rational Mech. Anal. 63 (1977), 337-403. MR 57:14788

[BM] Ball, J. M. and Murat, F., $W^{1, p}$-quasi-convexity and variational problems for multiple integrals, J. Funct. Anal. 58 (1984), 225-253. MR 87g:49011a

[G] J. B. Garnett, Bounded Analytic Functions, New York, Academic Press, 1970.

[HL] G. H. Hardy and J. E. Littlewood, Some properties of conjugate functions, J. Reine Angew. Math. 167 (1932), 405-423.

[I] T. Iwaniec, p-harmonic tensors and quasiregular mappings, Annals of Mathematics 136 (1992), 589-624. MR 94d:30034

[IL] T. Iwaniec and A. Lutoborski, Integral estimates for null Lagrangians, Arch. Rational Mech. Anal. 125 (1993), 25-79. MR 95c:58054

[IM] T. Iwaniec and G. Martin, Quasiregular mappings in even dimensions, Acta Math. 170 (1993), 29-81. MR 94m:30046

[IN] T. Iwaniec and C. A. Nolder, Hardy-Littlewood inequality for quasiregular mappings in certain domains in $R^{n}$, Ann. Acad. Sci. Fenn. Ser. A.I. Math. 10 (1985), 267-282. MR 87d:30022

[N1] C. A. Nolder, A characterization of certain measures using quasiconformal mappings, Proc. Amer. Math. Soc., Vol. 109, 2 (1990), 349-456. MR 90i:30034

[N2] C. A. Nolder, A quasiregular analogue of theorem of Hardy and Littlewood, Trans. Amer. Math. Soc., Vol. 3311 (1992), 215-226. MR 92g:30026

[N3] C. A. Nolder, Hardy-Littlewood theorems for A-harmonic tensors, Illinois J. Math., to appear.

[S] B. Stroffolini, On weakly A-harmonic tensors, Studia Math., 3114 (1995), 289-301. CMP $95: 14$

Department of Mathematics, Florida State University, Tallahassee, Florida 32306 3027

Current address: Department of Mathematics and Statistics, University of Minnesota, Duluth, Minnesota 55812-2496

E-mail address: sding@d.umn.edu 